\title{
Alteração da temperatura nos tecidos biológicos com a aplicação do ultrassom terapêutico: uma revisão
}

\author{
Temperature changes in biological tissue due to the \\ application of therapeutic ultrasound: a review
}

\author{
Daniela Akemi Itakura $^{[\mathrm{a}]}$, Viviane Magas $^{[\mathrm{b}]}$, Eduardo Borba Neves ${ }^{[\mathrm{c}]}$, Percy Nohama ${ }^{[\mathrm{d}]}$ \\ [a] Fisioterapeuta, mestranda em Tecnologia em Saúde, Pontifícia Universidade Católica do Paraná (PUCPR), Curitiba, PR - \\ Brasil, e-mail: ditakura@uol.com.br \\ [b] Fisioterapeuta, mestranda em Tecnologia em Saúde, Pontifícia Universidade Católica do Paraná (PUCPR), Curitiba, PR - \\ Brasil, e-mail: vivianemagas@hotmail.com \\ [c] Doutor em Engenharia Biomédica, Universidade Tecnológica Federal do Paraná (UTFPR), Curitiba, PR - Brasil, e-mail: \\ borbaneves@hotmail.com \\ [d] Doutor em Engenharia Biomédica, Pontifícia Universidade Católica do Paraná (PUCPR), Curitiba, PR - Brasil, e-mail \\ percy.nohama@gmail.com
}

\section{Resumo}

Introdução: 0 ultrassom (US) terapêutico atua nos tecidos biológicos por meio da vibração, que é capaz de produzir efeitos fisiológicos térmicos e não térmicos. Objetivo: Discutir os parâmetros que afetam o aquecimento dos tecidos biológicos, decorrente da aplicação do US. Materiais e métodos: Efetuou-se levantamento bibliográfico nas bases de pesquisa ISI WEB e SCOPUS. Foram selecionados os artigos, na língua inglesa, que tiveram como ponto central, o estudo da variação da temperatura após a aplicação do US em humanos. Resultados: Foram observadas variações significativas em relação ao aumento de temperatura tecidual. Para a frequência de $3 \mathrm{MHz}$, ocorre um aumento médio de $2,6^{\circ} \mathrm{C}$ nos tecidos superficiais; e para $1 \mathrm{MHz}$, há um aumento médio de $1,7^{\circ} \mathrm{C}$ em tecidos profundos. Há um maior aquecimento do tecido em relação ao tamanho da ERA (área de radiação efetiva), quanto menor o tamanho maior o aquecimento. Também há um aumento da temperatura em relação às intensidades. Intensidades variando entre 0,5 a $3 \mathrm{~W} / \mathrm{cm}^{2}$ apresentam um acréscimo significativo de temperatura. Em relação à forma de aplicação, o gel proporciona melhor acoplamento, gerando maior aquecimento com um tempo de aplicação de cinco minutos - o tempo 
de aplicação deve ser adequado à área de tratamento e à densidade de potência. Entretanto, a velocidade de aplicação parece não modificar o aquecimento até 7-8 cm/s, mesmo que dispositivos diferentes aqueçam em níveis diferentes. Conclusão: Os parâmetros para a aplicação do US devem ser controlados e ajustados para produzir o aquecimento profundo dos tecidos considerando a variação entre dispositivos.

Palavras-chave: Ultrassom terapêutico. Temperatura. Efeitos térmicos.

\section{Abstract}

Introduction: The therapeutic ultrasound act in biological tissues by means of vibration can produce physiological effects of thermal and non-thermal. Objective: To discuss the parameters that affect the heating of biological tissues by ultrasound. Materials and methods: We conducted a literature research based on ISI Web and SCOPUS database. We selected the articles in the English language that have studied the temperature variation after the application of ultrasound in humans. Results: There are significant variations related to tissue-temperature rise. For the $3 \mathrm{MHz}$ ultrasound, there is an average increase of $2.6{ }^{\circ} \mathrm{C}$ in superficial tissues, and to the $1 \mathrm{MHz} U \mathrm{~S}$, there is an average increase of $1.7^{\circ} \mathrm{C}$ in deep tissues. There is a higher heating of the treated tissue when an area twice the ERA (effective radiating area) size, the shorter the higher the heating, there is an increase in temperature relative intensities. Intensities ranging from 0.5 to $3 \mathrm{~W} / \mathrm{cm}^{2}$ have a significant increase in temperature. Regarding the mode of application, the gel provides an improved coupling, generating more heat with an application time of five minutes - the application time depends on the treatment area and power density. However, the speed of application appears to modify the heating up to 7-8 cm/s. Also, different commercial devices produce heat differently. Conclusion: Parameters for ultrasound application must be controlled and adjusted to generate deep heating of tissues considering that it may vary among devices.

Keywords: Therapeutic ultrasound. Temperature. Thermal effects.

\section{Introdução}

Por possuir ações físicas, biofísicas e terapêuticas, a energia acústica do ultrassom (US) é um recurso fisioterapêutico amplamente utilizado na prática clínica em diversas patologias. Por essas propriedades, desde sua introdução em processos terapêuticos há mais de 60 anos, o US tem sido objeto de estudo de muitos pesquisadores $(1,2,3)$.

Inicialmente, a aplicação terapêutica com US tinha a finalidade de promover aquecimento profundo dos tecidos, principalmente em lesões dos tecidos moles. Atualmente, o US tem sido utilizado para tratar patologias articulares e para acelerar o processo de cicatrização de feridas $(1,4)$.

Quando aplicado no corpo humano, as ondas ultrassônicas interagem com os tecidos biológicos e provocam alterações fisiológicas tradicionalmente divididas em efeitos térmicos e efeitos não térmicos. Na prática, entretanto, é extremamente difícil distingui-los, pois as interações mecânicas e térmicas ocorrem simultaneamente $(1,2)$. Neste estudo, serão examinados exclusivamente os efeitos térmicos do tratamento. Dentre os principais efeitos térmicos do US estão a redução da dor, aumento na perfusão, redução da rigidez articular, aumento na extensibilidade do colágeno e cicatrização tecidual $(1,3,5)$.

Cabe esclarecer, inicialmente, que, para que o US terapêutico produza o efeito térmico desejável, é necessário que atinja uma determinada temperatura no tecido alvo, entre 40 e $45^{\circ} \mathrm{C}$, por aproximadamente 5 min. (5). Se as temperaturas aplicadas ultrapassarem essa faixa, podem ocorrer danos celulares; e se as temperaturas forem inferiores a $40{ }^{\circ} \mathrm{C}$, os efeitos terapêuticos desejáveis podem não ser obtidos (6).

Starkey (7) relata os efeitos de acordo com o delta de elevação da temperatura: o aumento da temperatura em $1^{\circ} \mathrm{C}$ aumenta o metabolismo; o aquecimento de 2 a $3{ }^{\circ} \mathrm{C}$ diminui a dor e o espasmo muscular; e o aumento de $4{ }^{\circ} \mathrm{C}$ ou mais, aumenta a extensibilidade do colágeno e reduz a rigidez articular.

Tal extensão da resposta fisiológica ao calor depende de vários fatores: temperatura máxima atingida, taxa de elevação da temperatura, tempo de aquecimento e volume aquecido (1), área a ser tratada, frequência utilizada ( $1 \mathrm{MHz}$ ou $3 \mathrm{MHz}$ ), modo 
de emissão escolhido (contínuo ou pulsado), tempo de aplicação, intensidade utilizada no US (5) e especificidades do aparelho utilizado (8).

Kitchen (9) adverte que é preciso cautela ao tentar reproduzir em ambiente clínico os resultados obtidos em ambiente experimental porque as condições podem não ser equivalentes. Por essa razão, objetiva-se por este estudo fazer um levantamento dos principais trabalhos realizados em humanos no intento de analisar os fatores que afetam o aquecimento do tecido biológico gerado pela aplicação da onda ultrassônica.

\section{Materiais e métodos}

Inicialmente, procedeu-se a seleção dos artigos de pesquisa relevantes para este estudo. Foram coletados nas bases de dados ISI WEB e SCOPUS artigos produzidos no período de 1990 a 2010; esse recorte foi motivado pelo enfoque nos estudos experimentais do US em humanos ter iniciado na década de 90. Para a busca foram utilizadas as seguintes palavras-chave: temperature, therapeutic ultrasound e human. Optou-se pelas bases de dados ISI WEB OF SCIENCE e $S C O P U S$ por serem repositórios abrangentes e com indiscutíveis critérios de qualidade para ingresso de periódicos. Além disso, essas bases são largamente utilizadas por pesquisadores das áreas de saúde e tecnologia, para as quais o tema aqui abordado é expressivamente um de seus objetos de estudo.

Foram identificados 303 artigos publicados na língua inglesa sobre o US terapêutico. Os critérios de inclusão foram: abordam a aplicação do US exclusivamente em humanos; foram publicados em língua inglesa. Foram excluídos os estudos que não abordavam a temperatura em humanos (127 artigos), estudos que utilizaram os efeitos não térmicos do US (78 artigos) e os artigos duplicados (82 artigos). Por fim, foram analisados 16 artigos, nos quais buscou-se identificar os seguintes indicadores: autores, sítio anatômico, parâmetros utilizados no tratamento, população estudada, temperatura inicial, temperatura final, velocidade de aplicação, frequência, tamanho da área, modo de aplicação (contínuo e pulsado), intensidade, método de medida da temperatura, e duração do tratamento.

A análise dos dados foi realizada pela comparação dos diversos parâmetros utilizados na aplicação do US. A partir dos resultados encontrados, seria possível concluir quais os fatores implicam nos efeitos térmicos provocados pelo US.

\section{Resultados}

Foram encontrados 16 artigos que estudaram a variação da temperatura após a aplicação do US em humanos. Eles foram analisados e apresentados de forma resumida nos Quadros 1, 2 e 3. Os estudos foram agrupados pela frequência de operação do US: $3 \mathrm{MHz}, 1 \mathrm{Mz}$ ou a associação das duas frequências. Sob esses critérios, buscou-se mostrar o local de aplicação dos US, a profundidade da inserção do sensor para análise da variação de temperatura, as temperaturas intramusculares e tendíneas iniciais e finais, os parâmetros ultrassônicos utilizados e os resultados obtidos em cada um dos estudos.

Dentre os sete estudos que utilizaram o US de $3 \mathrm{MHz}$, os principais locais de aplicação foram o tríceps sural, gastrocnêmio e tendão patelar; nesses casos, a profundidade de inserção do sensor de temperatura variou de 1,2 a $2,5 \mathrm{~cm}$, de 1,6 a $2 \mathrm{~cm}$ e de $1 \mathrm{~cm}$, respectivamente.

Com referência às temperaturas com o US de $3 \mathrm{MHz}$, observou-se que a temperatura inicial variou de 33,8 a $35,5^{\circ} \mathrm{C}$ para o músculo tríceps sural, e de 35 a $35,5^{\circ} \mathrm{C}$ para o músculo gastrocnêmio; assim, observou-se variação a temperatura final conforme o objetivo do estudo e dos parâmetros utilizados (Quadro 1).

Em relação à análise dos parâmetros intensidade e área, observou-se que a intensidade variou de $1 \mathrm{a}$ $1,5 \mathrm{~W} / \mathrm{cm}^{2}$, modo contínuo, e a área de tratamento variou de duas a quatro vezes a ERA, sob um período de aplicação que variou entre 4 e 15 min.

Para o US de $1 \mathrm{MHz}$, os principais locais de aplicação foram o tríceps sural, nervo ulnar e região de L4-L5, com inserção do sensor de temperatura entre 3 a $5 \mathrm{~cm}$ de profundidade.

Em relação à temperatura, constatou-se que a temperatura inicial variou conforme a área de aplicação: de 35,4 a $38,1^{\circ} \mathrm{C}$ para o músculo tríceps sural; $32,7^{\circ} \mathrm{C}$ para o nervo ulnar; e $36,2^{\circ} \mathrm{C}$ na região de L4-L5. Ao final da aplicação do US, ocorreu elevação das temperaturas, com valores diferentes para cada estudo, como se observa no Quadro 2. Utilizando os parâmetros intensidade e tamanho da área de tratamento, nota-se variação de intensidade de 1 a 2,5 W/ $\mathrm{cm}^{2}$, no modo contínuo, com área de tratamento de 2 a 10 
vezes a ERA e período de aplicação de 5 a 10 minutos (Quadro 2).

Em relação aos dois estudos que compararam a aplicação dos US de 1 e 3 MHz, o local de aplicação foi o músculo tríceps sural. A profundidade de inserção do sensor de temperatura variou de 2,5 a 5 cm para o grupo de $1 \mathrm{MHz}$, e de 0,8 a 2,5 cm para o grupo de $3 \mathrm{MHz}$. Nesse contexto, a temperatura inicial variou de 35,3 a $35,5^{\circ} \mathrm{C}$ e a temperatura final variou de 36,6 a $40^{\circ} \mathrm{C}$. Em relação aos parâmetros utilizados, a intensidade variou de 0,5 a $2,0 \mathrm{~W} / \mathrm{cm}^{2}$, modo contínuo, área de tratamento 4 vezes a ERA, e tempo de aplicação de 7 a 10 minutos, como listado no Quadro 3.

Quadro 1 - Estudos que analisaram a produção do calor intramuscular e do calor do tendão com o US 3MHz

\begin{tabular}{|c|c|c|c|c|c|}
\hline Autor & $\begin{array}{l}\text { Local } \\
\text { (profundidade) }\end{array}$ & Temperatura inicial & Temperatura final & Resultados & Parâmetros \\
\hline Draper et al. (10) & Tríceps sural & Não relatado & Não relatado & $\begin{array}{l}0 \text { alongamento } \\
\text { com o US mostrou } \\
\text { um aumento } \\
\text { mais expressivo } \\
\text { na amplitude de } \\
\text { dorsiflexão do que o } \\
\text { alongamento isolado. }\end{array}$ & $\begin{array}{l}3 \mathrm{MHz}, 1,5 \mathrm{~W} / \mathrm{cm}^{2} \text {, } \\
\text { contínuo, } 7 \text { min., } \\
\text { área de tratamento } \\
4 \mathrm{ERA} \text {, velocidade } \\
4 \mathrm{~cm} / \mathrm{s} \text {, Omnisound } \\
\text { 3000C: 4,1ERA, } \\
\text { BNR } 3: 1\end{array}$ \\
\hline $\begin{array}{l}\text { Holcomb e Joyce } \\
\text { et al. (11) }\end{array}$ & $\begin{array}{l}\text { Tríceps sural } \\
(1,2 \mathrm{~cm})\end{array}$ & $\begin{array}{l}\text { Omnisound 3000C: } \\
34,7^{\circ} \mathrm{C} \\
\text { Forte } 400 \text { Combo: } \\
34,81^{\circ} \mathrm{C}\end{array}$ & $\begin{array}{l}\text { Omnisound } 3000 \mathrm{C} \text { : } \\
40,57^{\circ} \mathrm{C}\left(\uparrow 5,81^{\circ} \mathrm{C}\right) \\
\text { Forte } 400 \text { Combo: } \\
38,66^{\circ} \mathrm{C}\left(\uparrow 3,85^{\circ} \mathrm{C}\right)\end{array}$ & $\begin{array}{l}0 \text { Omnisound } \\
3000 \mathrm{C} \text { foi mais } \\
\text { eficaz em aumentar } \\
\text { a temperatura } \\
\text { intramuscular. }\end{array}$ & $\begin{array}{l}3 \mathrm{MHz}, 1,0 \mathrm{~W} / \mathrm{cm}^{2} \text {, } \\
\text { contínuo, } 10 \mathrm{~min} . \\
\text { área de tratamento } \\
\text { 2ERA, velocidade } \\
4 \mathrm{~cm} / \mathrm{s} \\
\text { Omnisound } 3000 \mathrm{C} \text { : } \\
\text { 4,9ERA, BNR 3,7:1 } \\
\text { Forte } 400 \text { Combo: } \\
\text { 4,6 ERA, BNR 2,3:1 }\end{array}$ \\
\hline Merrick et al. (5) & $\begin{array}{l}\text { Gastrocnêmio } \\
(1,6 \mathrm{~cm})\end{array}$ & $35^{\circ} \mathrm{C}$ a $35,5^{\circ} \mathrm{C}$ & $\begin{array}{l}\text { Omnisound 3000C: } \\
\uparrow 6^{\circ} \mathrm{C} \\
\text { Dynatron } 950 \text { : } \\
\uparrow 3,8^{\circ} \mathrm{C} \\
\text { Excel Ultra III: } \\
\uparrow 3,8^{\circ} \mathrm{C}\end{array}$ & $\begin{array}{l}\text { Há diferença } \\
\text { na elevação da } \\
\text { temperatura } \\
\text { intramuscular, } \\
\text { sendo maior com } \\
\text { o dispositivo } \\
\text { Omnisound } 3000 \mathrm{C} \\
\text { e semelhante entre } \\
\text { os outros dois. }\end{array}$ & $\begin{array}{l}3 \mathrm{MHz}, 1,5 \mathrm{~W} / \mathrm{cm}^{2} \text {, } \\
\text { contínuo, } 10 \mathrm{~min} \text {, } \\
\text { área de tratamento } \\
\text { 2ERA } \\
\text { Omnisound 3000C: } \\
\text { 6,7 ERA, BNR 3,9:1 } \\
\text { Dynatron 950: 5,0 } \\
\text { ERA, BNR < 6:1 } \\
\text { Excel Ultra III: } 5,0 \\
\text { ERA, BNR < 4:1 }\end{array}$ \\
\hline Gallo et al. (12) & $\begin{array}{l}\text { Gastrocnêmio } \\
(2 \mathrm{~cm})\end{array}$ & $35,3 \pm 0.7^{\circ} \mathrm{C}$ & $\begin{array}{l}38,1 \pm 0.6^{\circ} \mathrm{C} \\
\left(\uparrow 2,8 \pm 0,8^{\circ} \mathrm{C}\right)\end{array}$ & $\begin{array}{l}\text { O US pulsado } \\
\text { produziu } \\
\text { aumento similar } \\
\text { da temperatura } \\
\text { intramuscular ao } \\
\text { US contínuo. }\end{array}$ & $\begin{array}{l}3 \mathrm{MHz}, 10 \mathrm{~min} \text {, } \\
\text { área de tratamento } \\
5 \mathrm{~cm}^{2} \text { Omnisound } \\
3000 \mathrm{C}: 3,8 \mathrm{ERA} \text {, } \\
\text { BNR } 3,6: 1 \\
\text { Grupo } 1,0 \mathrm{~W} / \mathrm{cm}^{2} \text {, } \\
\text { pulsado } 50 \%, 10 \mathrm{~min} \\
\text { Grupo } 0,5 \mathrm{~W} / \mathrm{cm}^{2} \text {, } \\
\text { contínuo, } 10 \mathrm{~min} \text {. }\end{array}$ \\
\hline
\end{tabular}


Quadro 1 - Estudos que analisaram a produção do calor intramuscular e do calor do tendão com o US 3MHz

(Conclusão)

\begin{tabular}{|c|c|c|c|c|c|}
\hline Autor & $\begin{array}{l}\text { Local } \\
\text { (profundidade) }\end{array}$ & Temperatura inicial & Temperatura final & Resultados & Parâmetros \\
\hline Gulick et al. (13) & $\begin{array}{l}\text { Tríceps sural } \\
(2,5 \mathrm{~cm})\end{array}$ & $\begin{array}{l}\text { Grupo aquasonic } \\
\text { gel: } 35,5^{\circ} \mathrm{C} \\
\text { Grupo T-Prep: } \\
35,3^{\circ} \mathrm{C}\end{array}$ & $\begin{array}{l}37^{\circ} \mathrm{C}\left(\uparrow 1,5^{\circ} \mathrm{C}\right) \\
37,1^{\circ} \mathrm{C} \quad\left(\uparrow 1,8^{\circ} \mathrm{C}\right)\end{array}$ & $\begin{array}{l}\text { Não houve } \\
\text { diferença } \\
\text { estatisticamente } \\
\text { significante no } \\
\text { aquecimento entre } \\
\text { os grupos. }\end{array}$ & $\begin{array}{l}3 \mathrm{MHz}, 1,0 \mathrm{~W} / \mathrm{cm}^{2} \text {, } \\
\text { contínuo, } 15 \mathrm{~min} \text {, } \\
\text { área de tratamento } \\
6 \mathrm{~cm}^{2} \text {, velocidade } \\
3-4 \mathrm{~cm} / \mathrm{s} \text {, } \\
\text { Omnisound } 3000 \mathrm{C} \text { : } \\
2,1 \mathrm{ERA} \text {, BNR 2,9:1 }\end{array}$ \\
\hline $\begin{array}{l}\text { Draper e Ricard } \\
\text { et al. (14) }\end{array}$ & $\begin{array}{l}\text { Tríceps sural } \\
(1,2 \mathrm{~cm})\end{array}$ & $33,8 \pm 1,3^{\circ} \mathrm{C}$ & $39,1^{\circ} \mathrm{C} \pm 1,2^{\circ} \mathrm{C}$ & $\begin{array}{l}0 \text { alongamento } \\
\text { é mais efetivo } \\
\text { na janela de } \\
\text { tempo } 3,3 \text { min., } \\
\text { quando elevada } \\
\text { a temperatura } \\
\text { intramuscular } \\
\text { acima de } 5^{\circ} \mathrm{C} \text {. }\end{array}$ & $\begin{array}{l}3 \mathrm{MHz}, 1,5 \mathrm{~W} / \mathrm{cm}^{2} \text {, } \\
\text { contínuo, } 6 \mathrm{~min} \text {, } \\
\text { área de tratamento } \\
2 \times \text { ERA, velocidade } \\
4 \mathrm{~cm} / \mathrm{s} \text {, Omnisound } \\
3000 \mathrm{C}: 4,5 \mathrm{ERA} \text {, } \\
\text { BNR 1,8:1 }\end{array}$ \\
\hline Chan et al. (15) & $\begin{array}{l}\text { Tendão patelar } \\
(1 \mathrm{~cm})\end{array}$ & & $\begin{array}{l}\text { Grupo área de } \\
\text { tratamento } 2 \text { ERA: } \\
\uparrow 8,3^{\circ} \mathrm{C} \pm 1,7^{\circ} \mathrm{C} \\
\text { Grupo área de } \\
\text { tratamento } 4 \text { ERA: } \\
\uparrow 5,0^{\circ} \mathrm{C} \pm 1,0^{\circ} \mathrm{C}\end{array}$ & $\begin{array}{l}\text { Ambos os grupos } \\
\text { apresentaram } \\
\text { aumento na } \\
\text { temperatura, porém } \\
\text { de forma mais } \\
\text { significativa no } \\
\text { grupo } 2 \text { ERA. A taxa } \\
\text { de aquecimento por } \\
\text { minuto foi de } 2,1^{\circ} \mathrm{C} \\
\pm 0,4^{\circ} \mathrm{C} \text { (grupo } \\
2 \text { ERA) e de } 1,3^{\circ} \mathrm{C} \\
\pm 0,3^{\circ} \mathrm{C} \text { (grupo } 4 \\
\text { ERA). }\end{array}$ & $\begin{array}{l}3 \mathrm{MHz}, 1,0 \mathrm{~W} / \mathrm{cm}^{2} \text {, } \\
\text { contínuo, } 4 \mathrm{~min} \text {, } \\
\text { velocidade 2-3 } \\
\mathrm{cm} / \mathrm{s} \text {, Omnisound } \\
\text { 3000C: 4,5 ERA, } \\
\text { BNR 1,8:1 } \\
\text { Grupo área de } \\
\text { tratamento } 2 \text { ERA } \\
\text { Grupo área de } \\
\text { tratamento } 4 \text { ERA }\end{array}$ \\
\hline
\end{tabular}

Legenda: US = ultrassom; ERA = área de radiação efetiva; BNR = taxa de não uniformidade do raio.

Fonte: Dados da pesquisa.

Quadro 2 - Estudos que analisaram a produção de calor intramuscular com o US 1 MHz

(Continua)

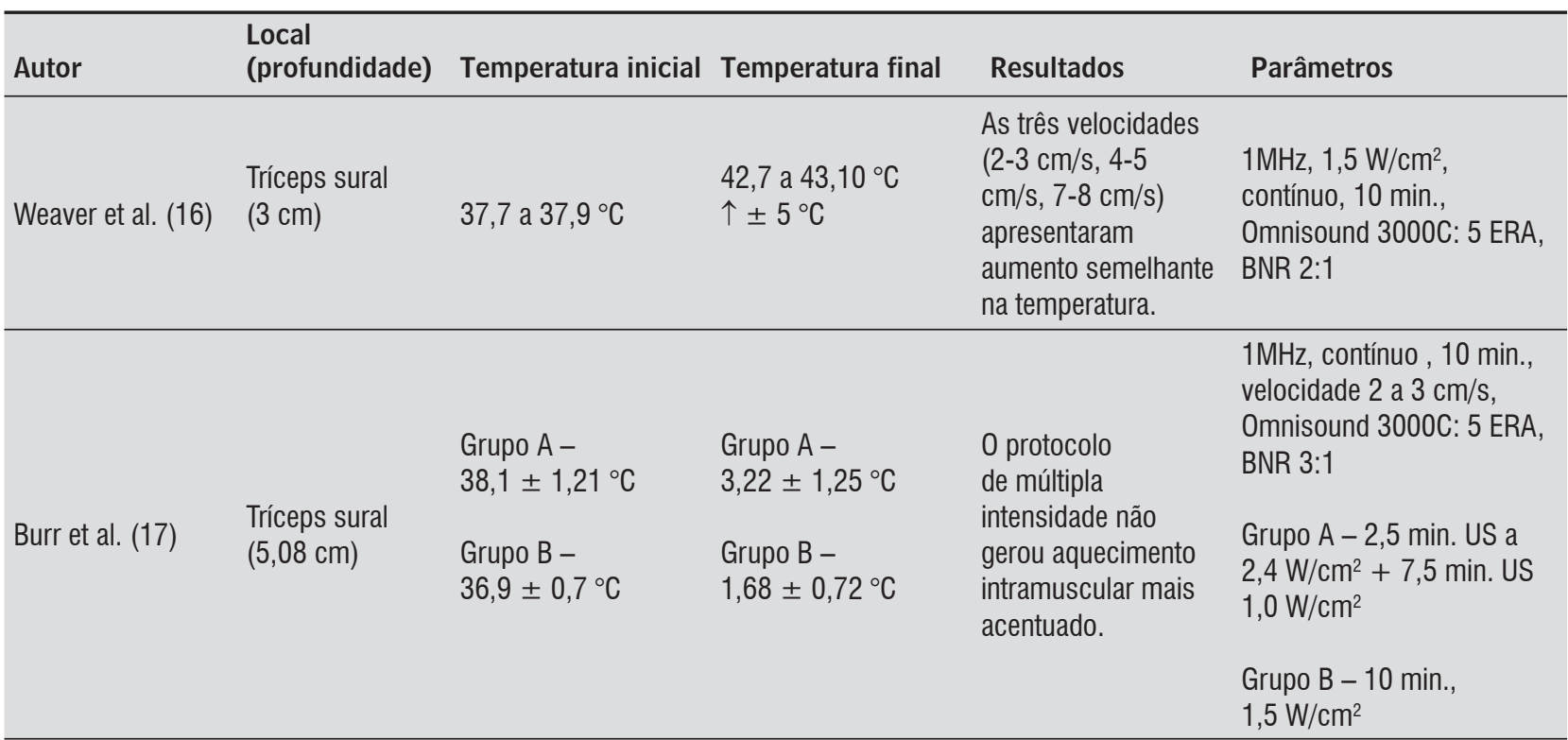


Quadro 2 - Estudos que analisaram a produção de calor intramuscular com o US 1 MHz

(Conclusão)

\begin{tabular}{|c|c|c|c|c|c|}
\hline Autor & $\begin{array}{l}\text { Local } \\
\text { (profundidade) }\end{array}$ & Temperatura inicial & Temperatura final & Resultados & Parâmetros \\
\hline $\begin{array}{l}\text { Leonard e Merrick } \\
\text { et al. (18) }\end{array}$ & $\begin{array}{l}\text { Tríceps sural } \\
(4 \mathrm{~cm})\end{array}$ & $\begin{array}{l}35,4 \pm 0,7^{\circ} \mathrm{C} \\
\text { Grupo } \mathrm{A}-35,5^{\circ} \mathrm{C} \\
\text { Grupo } \mathrm{B}-36^{\circ} \mathrm{C} \\
\text { Grupo } \mathrm{C}-35,8^{\circ} \mathrm{C} \\
\text { Grupo D }-35,4^{\circ} \mathrm{C}\end{array}$ & $\begin{array}{l}\text { Grupo } \mathrm{A}-36,3^{\circ} \mathrm{C} \\
\left(\uparrow 0,8^{\circ} \mathrm{C}\right) \\
\text { Grupo } \mathrm{B}-37^{\circ} \mathrm{C} \\
\left(\uparrow 1^{\circ} \mathrm{C}\right) \\
\text { Grupo } \mathrm{C}-36,6^{\circ} \mathrm{C} \\
\left(\uparrow 0,8^{\circ} \mathrm{C}\right) \\
\text { Grupo } \mathrm{D}-36^{\circ} \mathrm{C} \\
\left(\uparrow 0,6^{\circ} \mathrm{C}\right)\end{array}$ & $\begin{array}{l}\text { Nenhum tratamento } \\
\text { atingiu a elevação } \\
\text { da temperatura } \\
4^{\circ} \mathrm{C} \text {. } \\
1,0 \mathrm{~W} / \mathrm{cm}^{2} \text { aqueceu } \\
\text { mais do que a de } \\
2,0 \mathrm{~W} / \mathrm{cm}^{2} \text {. }\end{array}$ & $\begin{array}{l}1 \mathrm{MHz} \text {, contínuo, } 10 \mathrm{~min} \text {, } \\
\text { velocidade } 4 \mathrm{~cm} / \mathrm{s} \text {, Rich- } \\
\text { MarTheratouch: 4,5ERA, } \\
\text { BNR 5,5:1 } \\
\text { Grupo A: } 0,5 \mathrm{~W} / \mathrm{cm}^{2} \\
\text { Grupo B: } 1,0 \mathrm{~W} / \mathrm{cm}^{2} \\
\text { Grupo } \mathrm{C}: 1,5 \mathrm{~W} / \mathrm{cm}^{2} \\
\text { Grupo D: } 2,0 \mathrm{~W} / \mathrm{cm}^{2}\end{array}$ \\
\hline Frye et al. (19) & $\begin{array}{l}\text { Tríceps sural } \\
(5 \mathrm{~cm})\end{array}$ & $35,50^{\circ} \mathrm{C}$ & $40,40^{\circ} \mathrm{C}$ & $\begin{array}{l}\text { Aumento anormal } \\
\text { e amplo da taxa } \\
\text { de aquecimento } \\
\text { muscular } 0,4^{\circ} \mathrm{C} \text {. }\end{array}$ & $\begin{array}{l}1 \mathrm{MHz}, 1,5 \mathrm{~W} / \mathrm{cm}^{2} \text {, } \\
\text { contínuo, } 10 \mathrm{~min} \text {., área de } \\
\text { tratamento } 8,5 \mathrm{~cm} \text {. }\end{array}$ \\
\hline $\begin{array}{l}\text { Demchak et al. } \\
\text { (20) }\end{array}$ & $\begin{array}{l}\text { Tríceps sural } \\
(3 \mathrm{~cm})\end{array}$ & $370^{\circ} \mathrm{C}$ & $39,60^{\circ} \mathrm{C}$ & $\begin{array}{l}\text { Os três } \\
\text { transdutores } \\
\text { produziram } \\
\text { um aumento } \\
\text { significativo de } \\
\text { temperatura após } 1 \\
\text { min. de aplicação - } \\
0,2^{\circ} \mathrm{C} \text {. }\end{array}$ & $\begin{array}{l}1 \mathrm{MHz}, 1,2 \mathrm{~W} / \mathrm{cm}^{2} \text {, cont, } \\
10 \mathrm{~min}, 1,3 \mathrm{~W} / \mathrm{cm}^{2} \text {, } \\
1,4 \mathrm{~W} / \mathrm{cm}^{2} \text {, área de } \\
\text { tratamento } 4,4 \mathrm{~cm} \text {. }\end{array}$ \\
\hline Kramer (21) & $\begin{array}{l}\text { Antebraço } \\
\text { Nervo ulnar }\end{array}$ & $32,70^{\circ} \mathrm{C}$ & $33,00^{\circ} \mathrm{C}$ & $\begin{array}{l}\text { Imperceptível } \\
\text { mudança na } \\
\text { sensação de calor } \\
\text { de } 0,0 \mathrm{~W} / \mathrm{cm}^{2} \text { a } \\
1,5 \mathrm{~W} / \mathrm{cm}^{2} \text {. } \\
\\
\text { Na intensidade } \\
2,5 \mathrm{~W} / \mathrm{cm}^{2} \text {, percep- } \\
\text { ção de morno. }\end{array}$ & $\begin{array}{l}1 \mathrm{MHz} \text {, contínuo, } 5 \mathrm{~min} . \text {, } \\
\text { velocidade } 3 \mathrm{~cm} / \mathrm{s} \text {, área } \\
\text { de tratamento } 2 \mathrm{ERA} \text {, } \\
\text { gel transmissor, } \\
\text { intensidades: } \\
1,0 \mathrm{~W} / \mathrm{cm}^{2} \\
1,5 \mathrm{~W} / \mathrm{cm}^{2} \\
2,0 \mathrm{~W} / \mathrm{cm}^{2} \\
2,5 \mathrm{~W} / \mathrm{cm}^{2}\end{array}$ \\
\hline $\begin{array}{l}\text { Morissette et al. } \\
\text { (22) }\end{array}$ & L4- L5 & $36,20^{\circ} \mathrm{C}$ & $39,30^{\circ} \mathrm{C}$ & $\begin{array}{l}\text { As duas intensidades } \\
\left(1,5 \mathrm{~W} / \mathrm{cm}^{2} \mathrm{e}\right. \\
\left.2,0 \mathrm{~W} / \mathrm{cm}^{2}\right) \\
\text { produziram um } \\
\text { aumento rápido de } \\
\text { temperatura. } \\
\text { Em } 2,0 \mathrm{~W} / \mathrm{cm}^{2} \text {, } \\
\text { a temperatura } \\
\text { aumentou } \\
\text { bruscamente após } \\
6 \text { min. de aplicação } \\
\text { de US. }\end{array}$ & $\begin{array}{l}1,0 \mathrm{MHz}, 1,5 \mathrm{~W} / \mathrm{cm}^{2} \mathrm{e} \\
2,0 \mathrm{~W} / \mathrm{cm}^{2}, \text { contínuo, } 10 \\
\text { min., } 10 \text { ERA. }\end{array}$ \\
\hline
\end{tabular}

Legenda: US = ultrassom; ERA = área de radiação efetiva; BNR = taxa de não uniformidade do raio.

Fonte: Dados da pesquisa. 
Quadro 3 - Estudos que analisaram a produção de calor com o US 1 MHz e 3 MHz

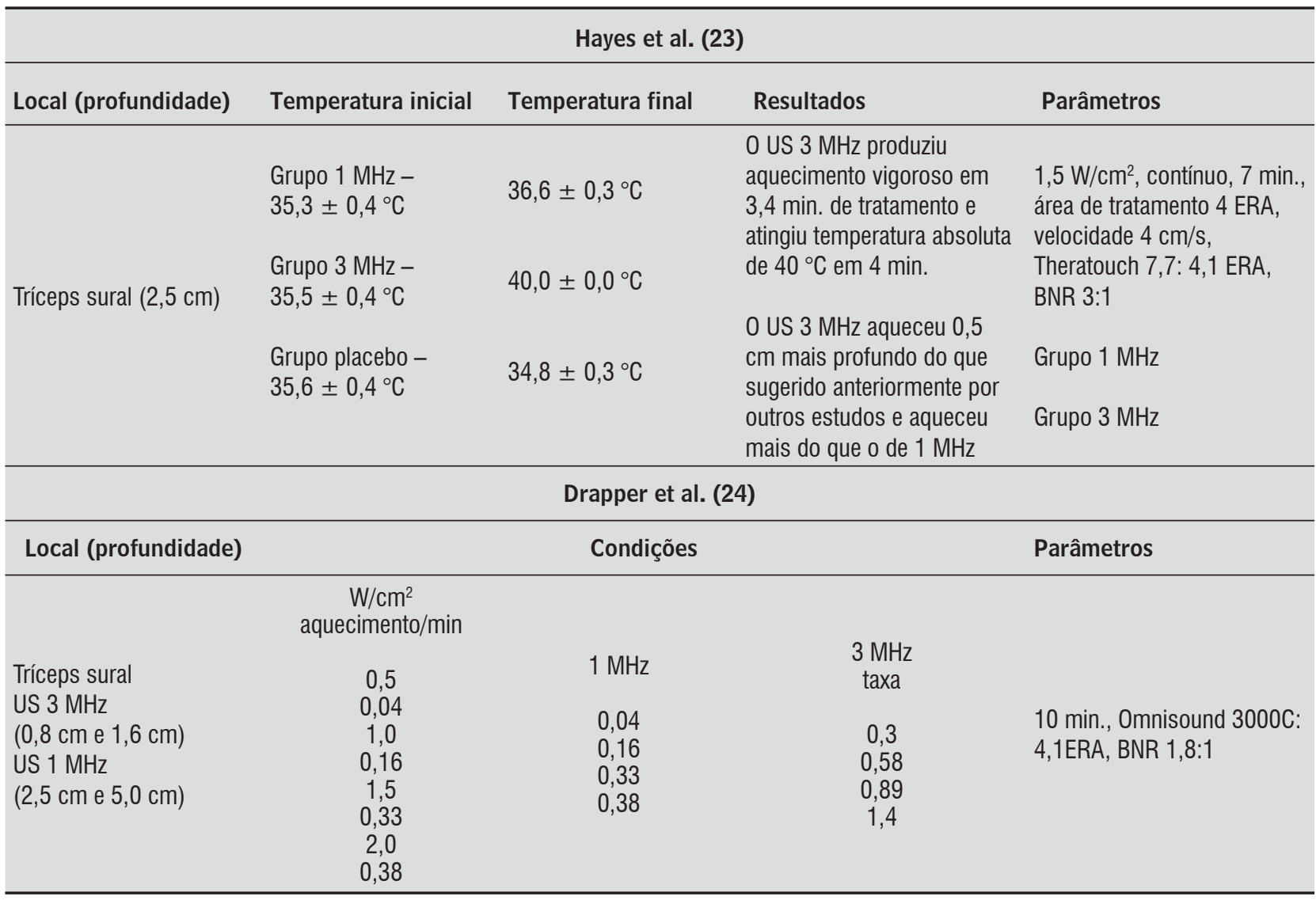

Legenda: US = ultrassom; ERA = área de radiação efetiva; BNR = taxa de não uniformidade do raio.

Fonte: Dados da pesquisa.

\section{Discussão}

No presente artigo, foram avaliados os parâmetros utilizados para quantificar o efeito térmico do tratamento com US a partir da análise de artigos que verificaram a produção de calor intramuscular com US de 1 e 3 MHz.

Com relação aos parâmetros utilizados (intensidade, tamanho da área, modo e tempo de aplicação), avaliaram-se os artigos que analisaram quantitativamente as alterações de temperaturas entre os US.

0 efeito térmico, sobre temperatura muscular de base entre 36 e $37^{\circ} \mathrm{C}$, pode ser dividido em três categorias: leve $\left(1{ }^{\circ} \mathrm{C}\right)$, moderado $\left(2-3{ }^{\circ} \mathrm{C}\right)$ e vigoroso $\left(>4^{\circ} \mathrm{C}\right) .0$ aquecimento leve está relacionado com a diminuição da inflamação subaguda e aceleração da taxa metabólica. 0 aquecimento moderado tem o objetivo de atuar no tratamento da inflamação crônica, dor e espasmo muscular, além de aumentar o fluxo sanguíneo. Já o aquecimento vigoroso é aplicado para aumentar a extensibilidade do colágeno e diminuir a rigidez articular $(7,25)$.

Cabe advertir, porém, que é problemático o uso dessa mudança relativa da temperatura basal como uma diretriz de tratamento porque não há uniformidade entre as temperaturas iniciais. Isso foi constatado nesta revisão a partir da observação de que a temperatura intramuscular é bastante variável, como mostrado nos Quadros 1, 2 e 3 (variando de 33,8 a $35,5^{\circ} \mathrm{C}$, em profundidades menores que $2,5 \mathrm{~cm}$, e de 35,5 a $38,1^{\circ} \mathrm{C}$ em profundidades entre 2,5 e $5 \mathrm{~cm}$ ). Essa constatação considera o tecido examinado e a profundidade, além da variabilidade intrínseca entre os seres humanos.

É importante lembrar que, embora seja capaz de produzir inúmeros efeitos benéficos nos estágios subsequentes, o aquecimento pode ser prejudicial ao reparo dos tecidos nos estágios iniciais, uma vez que esse procedimento pode aumentar o sangramento, o edema e a atividade metabólica, acarretando 
também aumento da dor (9). Além disso, outros estudos sugerem que temperaturas acima de $45^{\circ} \mathrm{C}$ são potencialmente danosas aos tecidos $(5,7,23)$.

Para obter tais temperaturas na camada de tecido mais profunda, vários parâmetros ultrassônicos podem ser ajustados, tais como: intensidade, frequência, modo de transferência de energia (contínuo ou pulsado), protocolo de tratamento (estático ou dinâmico), tipo de tecido a ser tratado e o próprio equipamento usado $(6,26)$.

\section{Fatores relacionados aos parâmetros}

de tratamento

\section{Frequência}

A frequência é selecionada com base na profundidade do tecido a ser tratado (7). Relata-se que o coeficiente de absorção varia linearmente com a frequência e que a taxa de deposição da energia aumenta com a diminuição da frequência a uma intensidade fixa. Assim, o US de $3 \mathrm{MHz}$ é usado para aquecer estruturas mais superficiais $(<2,5 \mathrm{~cm}$ de profundidade) como os tendões, ao passo que o US de $1 \mathrm{MHz}$ é usado para aquecer estruturas mais profundas $(2,5-5 \mathrm{~cm}$ de profundidade), como os músculos $(7,9)$.

0 estudo realizado por Drapper et al. (24) revelou que o US de $1 \mathrm{MHz}$ apresentou um aumento da taxa de aquecimento mais lento do que o US de $3 \mathrm{MHz}$ (Quadro 3).

Velocidade do movimento do transdutor

Durante a aplicação do US, recomenda-se mover o transdutor e não realizar a aplicação estática devido ao risco de causar dor e queimadura, baseando-se na taxa de não uniformidade do raio (BNR). A BNR é uma representação da homogeneidade da transmissão de energia, e pode ser responsável pela variabilidade no efeito do aquecimento entre aparelhos (7).

A maioria dos estudos que analisam a temperatura intramuscular com a aplicação do US em seus protocolos experimentais controlam a velocidade de aplicação do transdutor e recomendam entre 2 e $4 \mathrm{~cm} / \mathrm{s}$, como mostrado nos Quadros 1, 2 e 3. A justificativa é que a velocidade maior do que $4 \mathrm{~cm} / \mathrm{s}$ implicaria em diminuição da quantidade de energia absorvida e levaria à diminuição dos benefícios do aquecimento $(7,27,28)$.
Entretanto, Weaver et al. (16), ao analisarem o efeito da velocidade de aplicação do transdutor sobre a temperatura intramuscular em três diferentes velocidades $(2-3 \mathrm{~cm} / \mathrm{s}, 4-5 \mathrm{~cm} / \mathrm{s}, 7-8 \mathrm{~cm} / \mathrm{s})$, constataram que houve aumento similar da temperatura durante $o$ tratamento, nas três velocidades estudadas, $\operatorname{com} 1 \mathrm{MHz}$, $1,5 \mathrm{~W} / \mathrm{cm}^{2}$, no modo contínuo, durante dez minutos. Seria possível inferir, portanto, que a velocidade de aplicação não interfere no aquecimento intramuscular.

\section{Área de aplicação}

A área de aplicação tem relação direta com o tamanho da cabeça do transdutor ultrassônico. Para potencializar a eficácia do procedimento, deve-se determinar a área de tratamento em relação à área de radiação efetiva (ERA) da cabeça do transdutor do US, lembrando que a ERA é a área para a qual a energia é conduzida e que muitas vezes é menor do que o tamanho da cabeça do transdutor ultrassônico.

Estudo realizados (15) - nos quais foram utilizados termistores para registrar as alterações na temperatura com a administração do US de $3 \mathrm{MHz}$, com uma densidade de $1 \mathrm{~W} / \mathrm{cm}^{2}$, variando a ERA -, verificou-se que o aquecimento do tendão patelar era maior quando a área tratada correspondia a duas vezes a ERA do que quando a área de tratamento tinha quatro vezes o tamanho da ERA. Isso aponta a importância da escolha do transdutor de acordo com o tamanho da área a ser tratada.

Modo de aplicação (contínuo versus pulsado)

A energia acústica pode ser aplicada de forma contínua ou pulsada. No modo contínuo, a onda ultrassônica é continuamente gerada durante toda a aplicação e a energia é produzida em $100 \%$ do tempo. Já no modo pulsado, a intensidade é periodicamente interrompida e nenhuma energia é produzida durante o período desligado $(7,28)$. 0 modo contínuo está associado aos efeitos térmicos, ao passo que o modo pulsado se associa aos efeitos não térmicos. Apesar dos diferentes mecanismos de ação, Haar (1) e Baker et al. (2) relatam que não é possível separar os dois efeitos.

Com exceção do estudo realizado por Galo et al. (12), os estudos analisados nesta revisão de literatura utilizaram o modo contínuo (Quadros 1, 2 e 3). Na pesquisa de Galo et. al. (12), os autores compa- 
raram a aplicação do US de $3 \mathrm{MHz}$ em dois modos diferentes, um grupo no modo contínuo $0,5 \mathrm{~W} / \mathrm{cm}^{2} \mathrm{e}$ outro grupo no modo pulsado $1,0 \mathrm{~W} / \mathrm{cm}^{2}$, com $50 \%$ de ciclo de trabalho, durante 10 minutos. Nesse contexto, não foram identificadas diferenças no aumento da temperatura entre os dois grupos. Esse resultado sugere que, com a variação de intensidade, apesar de o modo de aplicação privilegiar um determinado tipo de efeito (térmico ou não térmico), é possível obter efeitos semelhantes com modos de aplicação diferentes (contínua e pulsada).

\section{Intensidade}

Um dos fatores que influenciam o aumento de temperatura nos tecidos produzido pelas ondas de US é a intensidade de saída. A densidade de potência, dada em $\mathrm{W} / \mathrm{cm}^{2}$, representa a potência liberada por unidade de área (29).

Em equipamentos tradicionais, a densidade de potência varia de 0,75 a $3 \mathrm{~W} / \mathrm{cm}^{2}$. Kitchen (8) relata que os efeitos terapêuticos do US ocorrem entre 0,5 e $3 \mathrm{~W} / \mathrm{cm}^{2}$, havendo sempre a produção de calor nas densidades mais elevadas.

A intensidade selecionada é determinada pela profundidade dos tecidos, a frequência da onda e o período de aplicação, cujo objetivo é a produção do efeito térmico, considerado de grande importância (30). Ao ajustar a intensidade em um nível muito baixo, o efeito terapêutico é nulo; por outro lado, se a intensidade for muito elevada, provocará efeitos indesejáveis como a lesão do tecido (29).

\section{Procedimento de aplicação}

Para que a onda ultrassônica atinja o tecido biológico, é imprescindível eliminar a camada de ar existente entre o transdutor e a pele do paciente porque $o$ ar reflete quase toda a onda emitida. Isso é alcançado usando agentes de acoplamento. Segundo Fuirini e Longo (29), a transferência de energia poderá ocorrer de duas formas: no contato direto entre o cabeçote e o corpo ou pela aplicação submersa.

0 emprego de um meio de acoplamento visa facilitar a transferência da energia ultrassônica aos tecidos. A habilidade de um meio de acoplamento em transmitir as ondas ultrassônicas define sua eficácia. Então, se o aquecimento do tecido é o resultado do tratamento desejado, é fundamental eleger um meio de acoplamento que favoreça esse processo (13).

Os agentes de acoplamento devem ter certas características, tais como: impedância, coeficiente de reflexão, transmissão e atenuação. Os agentes de acoplamento mais usados na técnica de contato direto são: gel, óleo mineral e água, que são usados na técnica de imersão (31). Superfícies irregulares, como as extremidades distais, dificultam o contato entre o cabeçote e a pele. Nesse caso, utiliza-se a técnica de imersão, que proporciona uma dose de radiação ultrassônica mais uniforme $(7,28)$.

0 modo de transferência de energia por contato direto com gel é o mais utilizado. 0 transdutor, coberto por uma fina camada de gel, é colocado diretamente sobre a pele, buscando o contato de toda superfície do transdutor com a pele. Por diminuir a resistência da transmissão de onda, o gel evita a presença de bolhas de ar, aumenta o calor no tecido-alvo e minimiza sua dissipação $(7,8)$.

\section{Duração do tratamento}

A duração do tratamento é determinada pela intensidade de saída, pelas especificidades do tratamento e pela área a ser tratada $(7,28) .0$ aumento da temperatura durante a aplicação do US está relacionado ao tempo de aplicação e à intensidade de tratamento (7).

Os textos revisados expressam diferentes conclusões sobre a duração do tratamento; porém, todas preconizam que a duração depende da dimensão da área a ser tratada e da intensidade. Furini e Longo (29) recomendam que seja dispendido um período de tratamento total máximo de 15 minutos. Segundo Guirro e Guirro (30), estabelece-se, como regra geral, o tempo de 2 minutos para áreas próximas de $10 \mathrm{~cm}^{2}$.

Fatores relacionados ao local de aplicação e ao equipamento

A extensão em que um tecido absorve a energia de uma onda ultrassônica varia conforme o tipo de tecido. Os tecidos com alto teor proteico - como músculo e tendão - absorvem mais essa energia $(7,28)$. A maioria dos estudos analisados aplicaram o US no músculo tríceps sural (Quadros 1, 2 e 3).

Johns et al. (32) demonstraram que o efeito do aquecimento pelo US varia entre os equipamentos produzidos pelo mesmo fabricante. Além disso, 
provaram que a intensidade espacial média (SAI) medida era diferente da relatada pelo fabricante, exceto em um de seis equipamentos testados. Demchak et al. (33) relataram que a taxa do aquecimento não é linear como outrora pensado e que, por essa razão, varia entre os transdutores produzidos pelo mesmo fabricante.

Diferenças na variação de temperatura têm sido relatadas com diferentes transdutores de US, apesar de serem usados os mesmos parâmetros de tratamento. Os estudos realizados $(5,11)$, que avaliaram diferentes equipamentos de US, constataram discrepâncias no aumento de temperatura e na taxa de aquecimento. Essa diferença foi de aproximadamente $2{ }^{\circ} \mathrm{C}$ entre o que apresentou maior aquecimento e o menor aquecimento. Além disso, pode haver diferenças na taxa de aumento de temperatura entre transdutores de US de um mesmo fabricante (27).

Considerando que a variabilidade de aquecimento entre diferentes transdutores de US é bem conhecida, estudiosos têm sinalizado que as variações na SAI podem ser responsáveis pela inconsistência observada nos resultados dos pacientes. A SAI pode ser calculada dividindo-se a potência (W) do US pela área de radiação efetiva (ERA) do cabeçote. Portanto, discrepâncias na SAI dependem das variações na ERA e da energia de saída empregada $(34,32,35)$.

A variação de aquecimento apresentada entre diferentes equipamentos de US pode ter ocorrido pelo não cumprimento da normatização internacional. A International Electrotechnical Commission (IEC) apresenta requisitos particulares para a segurança do equipamento de US. A norma IEC (36) sugere que as variações de temperatura estão relacionadas com a energia de saída incorreta (36).

Segundo a IEC, a intensidade máxima eficaz não deve exceder $3 \mathrm{~W} / \mathrm{cm}^{2}$ e a potência de saída não pode ser reduzida mais que $5 \%$ da potência nominal. 0 feixe irradiado dever ser homogêneo e estável em relação ao tempo de aplicação. Alterações de temperatura em uma mesma área possivelmente são ocasionadas por um problema com o feixe de radiação. Cabe o esclarecimento de que nenhum dos estudos analisados avaliou a distribuição do feixe de radiação dos aparelhos utilizados.

\section{Conclusão}

Os parâmetros de tratamento usados para produzir aquecimento dos tecidos têm sido descritos detalhadamente na literatura. Parece haver um consenso em relação aos parâmetros que deveriam ser usados para provocar os efeitos térmicos no músculo, mas há inconsistência desse efeito produzido pelos diferentes equipamentos com um mesmo ajuste de potência ou energia de saída. Então, os resultados produzidos clinicamente por um aparelho de US podem ser diferentes daqueles obtidos por outros tipos de equipamentos US, ainda que utilizados parâmetros idênticos para o procedimento.

Como observado neste estudo, para obter aquecimento profundo existem inúmeras variáveis a serem controladas, mesmo quando o procedimento é realizado em laboratórios de pesquisa (onde é feita a calibragem dos aparelhos e o controle da área de tratamento).

Todos os estudos aqui analisados mediram a variação da temperatura com um sensor (do tipo termistor) intramuscular, que é um procedimento invasivo e inviável na prática clínica. No entanto, os estudos que visam avaliar os benefícios terapêuticos do US devem associar essa metodologia de controle da temperatura para justificar seus efeitos terapêuticos positivos ou não; isso porque, muitas vezes, a temperatura pode não atingir o valor ideal, o que, por conseguinte, influencia o resultado terapêutico.

Atualmente, tem sido indicado o uso da termografia - uma técnica que fornece um mapeamento térmico da superfície irradiada por US - para medir e monitorizar as variações térmicas produzidas pelo US.

\section{Referências}

1. Haar GT. Therapeutic ultrasound. Eur J Ultrasound. 1999;9:3-9. doi:10.1016/S0929-8266(99)00013-0.

2. Baker KG, Robertson VJ, Duck FA. A review of therapeutic ultrasound: biophysical effects. Phys Ther. 2001;81(7):1351-8. PMid:11444998.

3. Speed CA. Therapeutic ultrasound in soft tissue lesions. Rheumatology. 2001;40(12):1331-6. doi:10.1093/rheumatology/40.12.1331.

4. Wong RA, Schumann B, Townsend R, Phelps CA. A survey of therapeutic ultrasound use by physical therapists who are orthopaedic certified specialists. Phys Ther. 2007;87(8):986-94. doi:10.2522/ptj.20050392.

5. Merrick MA, Bernard KD, Devor ST, Williams JM. Identical 3-MHz ultrasound treatments with different devices produce different intramuscular temperatures. J Orthop Sports Phys Ther. 2003;33(7):379-85. PMid:12918863. 
6. Maggi LE, Omena TP, von Kruger MA, Pereira WCA. Software didático para modelagem do padrão de aquecimento dos tecidos irradiados por ultra-som fisioterapêutico. Rev Bras Fisioter. 2008;12(3):20414. doi:10.1590/S1413-35552008000300008.

7. Starkey C. Recursos terapêuticos em fisioterapia. 2. ed. São Paulo: Manole; 2001.

8. Kitchen S, Bazin S. Eletroterapia de Clayton. São Paulo: Manole; 1996.

9. Kitchen S. Eletroterapia: prática baseada em evidência. São Paulo: Manole; 2003.

10. Draper DO, Anderson C, Schulthies SS, Ricard MD. Immediate and residual change in dorsiflexion range of motion using an ultrasound heat and stretch routine. J Athl Train. 1998;33(2):141-4. PMid:16558501.

11. Holcomb WR, Joyce CJ. A comparison of temperature increases produced by 2 commonly used ultrasound units. J Athl Train. 2003;38(1):24-7. PMid:12937468

12. Gallo JA, Draper DO, Fellingham WG, Brad TL. A Comparison of human muscle temperature increases during 3-mhz continuous and pulsed ultrasound with equivalent temporal average intensities. J Orthop Sports Phys Ther. 2004;34(7):395-401. PMid:15296367.

13. Gulick DT, Ingram N, Krammes T, Wilds C. Comparison of tissue heating using $3 \mathrm{MHz}$ ultrasound with T-Prep versus Aquasonic gel. Phys Ther Sport. 2005; 6(1):131-6. doi:10.1016/j.ptsp.2005.04.001.

14. Draper DO, Ricard MD. Rate of temperature decay in human muscle following $3 \mathrm{MHz}$ ultrasound: the stretching window revealed. J Athl Train. 1995;30(4):3047. PMid:16558352.

15. Chan AK, Myrer JW, Measom GJ, Draper DO. Temperature changes in human patellar tendon in response to therapeutic ultrasound. J Athl Train. 1998; 33(2):130-5. PMid:16558499.

16. Weaver SL, Demchak TJ, Stone MB, Bracker O, Burr P. Effect of transducer velocity on intramuscular temperature during a $1 \mathrm{MHz}$ ultrasound treatment. J Orthop Sports Phys Ther. 2006;36(5):320-5. PMid:16715832.

17. Burr PO, Demchak TJ, Cordova ML, Ingersoll CD, Stone MB. Effects of altering intensity during $1-\mathrm{MHz}$ Ultrasound treatment on increasing triceps surae temperature. J Sport Rehabil. 2004;13:275-86.
18. Leonard J, Merrick M. A Comparison of intramuscular temperatures during 10-minute 1.0-mhz ultrasound treatments at different intensities. J Sport Rehabil. 2004;13:244-54.

19. Frye JL, Johns LD, Tom JA, Ingersoll CD. Blisters on the anterior shin in 3 research subjects after a 1-MHz, 1.5$\mathrm{W} / \mathrm{cm}^{2}$, continuous ultrasound treatment: a case series. J Athl Train. 2007;42(3):425-30. PMid:18060000.

20. Demchak JT, Straub SJ, Johns LD. Ultrasound heating is cuvilinear in nature and varies between transducers from the same manufacturer. J Sport Rehabil. 2007;16:122-30. PMid:17918699.

21. Kramer FJ. Sensory and motor nerve conduction velocities following therapeutic ultrasound. Aust J Physiother. 1997;33:245-50.

22. Morisette CD, Brown D, Saladin EM. Temperature change in lumbar periarticular tissue with cotinuous ultrasound. J Orthop Sports Phys Ther. 2004;34(12):250-5.

23. Hayes BT, Merrick MA, Sandrey MA, Cordova ML. Three-MHz ultrasound heats deeper into the tissues than originally theorized. J Athl Train. 2004;39:230-4. PMid:15496991.

24. Draper DO, Castel C, Castle D. Rate of temperature increase in human muscle during $1 \mathrm{mhz}$ and $3 \mathrm{mhz}$ continuous ultrasound. JOSPT. 1995;22:142-50. PMid:8535471.

25. Harr GT. Therapeutic applications of ultrasound. Prog Biophys Mol Biol. 2007;93:111-29. doi:10.1016/j. pbiomolbio. 2006.07.005.

26. Demmink JH, Helders PJ, Hobaek H, Enwemeka C. The variation of heating depth with therapeutic ultrasound frequency in physiotherapy. Ultrasound in Med \& Biol. 2003;29:113-8. doi:10.1016/ S0301-5629(02)00691-9.

27. Rubley MD, Touton TM. Thermal ultrasound: it's more than power and time. Human Kinetics. 2009;14:5-8.

28. Low J, Reed A. Eletroterapia explicada: princípios e prática. São Paulo: Manole; 2001.

29. Furini NJ, Longo GJ. Ultra-som. KLD - Biossistemas equipamentos eletrônicos. São Paulo: Amparo; 1996.

30. Guirro E, Guirro R. As variáveis físicas do ultra-som terapêutico: uma revisão. Rev Ciência \& Tecnol. 1996;9(5):31-41. 
31. Casarotto RA, Adamowski JC, Fallopa F, Bacanelli, F. Coupling agents in therapeutic ultrasound: acoustic and thermal behavior. Archives Physical Medicine and Rehabilitation. 2004;85(1):162-5. doi:10.1016/ S0003-9993(03)00293-4.

32. Johns LD, Straub S, Howard SM. Analysis of effective radiating area, power, intensity, and field characteristics of ultrasound transducers. Phys Med Rehabil. 2007;88:124-9. doi:10.1016/j.apmr.2006.09.016.

33. Demchak JT, Sotne BM. Effectiveness of clinical ultrasound parameters on changing intramuscular temperature. J Sport Rehabil. 2008;17(3):220-9. PMid:18708676.

34. Straub SJ, Johns LD, Howard SM. Variability in effective radiating area at $1 \mathrm{MHz}$ affects ultrasound treatment intensity. Phys Ther. 2008;88(1):50-7. doi:10.2522/ ptj.20060358.
35. Prentice WE. Modalidade terapêutica para fisioterapeutas. Porto Alegre: Artmed; 2003.

36. International Electrotechnical Commission. Particular requirements for the safety of ultrasonic physioterapy equipament; 2000. (part 2-5).

Recebido: 16/05/2011 Received: 05/16/2011

Aprovado: 08/12/2011

Approved: 12/08/2011 